\title{
People power
}

\author{
A wealth of potential exists for citizen science to contribute to major ecological and societal challenges. We can all \\ play a part by contributing to these projects, and encouraging our networks to do so too.
}

l: n the 2019 podcast drama 'Forest 404', the lead character listens to the sound of a rainforest for the very first time, as if it were some alien world. The play is set in the twenty-fourth century when a cataclysmic event has wiped out these ecosystems and digital audio archives are all that remain.

However, thanks to citizen science, an appreciation of ecological soundscapes is not only the preserve of fiction. Volunteer-assisted projects such as Soundscapes to Landscapes deploy acoustic recorders for monitoring biodiversity, and FrogID aims to establish a nationwide database of frog calls recorded on people's smartphones. Less than a year into the current global pandemic, researchers have also now established through acoustic monitoring of bird calls in San Francisco (United States), that reductions in road traffic noise during the recent state-wide COVID-19 shutdown had rapid knock-on impacts on song patterns of wild white-crowned sparrows ${ }^{1}$.

The societal upheaval that accompanied this global crisis has caused many of us who live in cities to re-assess our relationship with the natural world - either passively through increased time spent in home gardens or nearby parks, or by actively seeking out the positive effect that greenspaces can have on mental and physical well-being. Birdwatching and nature engagement have surged, and the global 'anthropause' has increased our awareness of the degree to which human behaviour and society can affect wildlife ${ }^{2}$.

This acute change in our relationship with biodiversity has come against the backdrop of a boom in technology, allowing people to engage more deeply with it, and at the same time contribute to big ecological data. Platforms such as iNaturalist are now using artificial-intelligence-assisted technology to bring automated species-identification apps to smartphones, allowing georeferenced occurrence records from anywhere in the world to be linked directly to databases such as the Global Biodiversity Information Facility. At the time of writing, the platform had surpassed 50 million observations of plants, animals and fungi, with coverage in every country on Earth. Its highest number of records was posted in the early stages of the pandemic in May 2020, at more than 3 million observations submitted worldwide.

Data available at these kinds of scales would have been unimaginable just a few years ago, and indeed other citizen science programmes, such as the more taxon-specific eBird platform, now contain more than 800 million observations since it launched in 2002.

The ubiquity of smartphones means that technology such as this can provide multiple wins for ecologists and conservation biologists. Data collected on this scale have potential to fill the well-known geographic biases in biodiversity databases - particularly in biodiverse regions of the Global South ${ }^{3}$. It also provides an opportunity for non-specialists to engage with the natural world like never before. For example, a companion app developed by iNaturalist called Seek provides a simplified, family-friendly version of the platform in which users can identify species in real time using their smartphone camera.

These engaging, intuitive guides are powerful tools in providing a gateway to a deeper understanding and appreciation of the natural world for novices. There is immense value in being able to name the organisms that surround you, and in doing so nature becomes less remote and removed from everyday experience, even in highly urbanized environments ${ }^{4}$. Access to natural history knowledge has never been more straightforward and accessible, and has the power to reverse concerns that we have become dissociated from nature in recent decades. There are also real opportunities with technologies such as this to make progress on equity and inclusion by lowering barriers to participation for underrepresented groups in these traditionally white-dominated spaces ${ }^{5,6}$. These approaches could also readily be incorporated into teaching at all stages of education to provide research grade data on a vast scale ${ }^{7,8}$.

As scientists and citizens, we can all support the growth and development of this approach by ensuring that we remain engaged ourselves, using the technology on fieldwork, in courses, in our leisure time and encouraging our networks of friends and family to do the same.

In terms of basic and applied ecology, citizen science data provide a wealth of potential that could also be exploited while fieldwork is cancelled or postponed during the current pandemic. New species have been identified formally, and those once thought lost to science have been confirmed alive and well via smartphone apps. The geographic extent of users engaged in citizen science projects means that it could be used as a sentinel of invasive species monitoring ${ }^{9}$, as well as provide a useful framework for conservation actions involving migratory species ${ }^{10}$. Platforms that integrate not only occurrence data but also phenotypic data from photographs, also offer new opportunities for targeted studies on individual species, by gathering more data on intraspecific variation than would likely ever be possible in a single field campaign. As a recent example, Moore et al. were able to extract range-wide georeferenced images of dragonflies from iNaturalist to explore the interplay between sexually selected wing colouration and climate ${ }^{11}$.

These examples represent just a selection of the possible questions that could be explored in fields such as phenology, biogeography, macroecology, conservation and evolution. A major challenge for citizen science developers will now be to ensure that these records are linked to broader information for users on why biodiversity matters, hopefully avoiding the prospect that they become only digital records of ecosystems declines, like the lost audio archives in Forest 404.

Citizen science is a broad term, and projects come in a huge diversity of different forms and approaches that may not only be restricted to data collection ${ }^{12}$. Where data collection is a primary focus, this scale and variety means that there is huge potential to make meaningful contributions to complex interlinked societal challenges like the United Nations (UN) Sustainable Development Goals, especially filling gaps in indicator data that have come mainly from traditional governmental or international organizations $^{13}$. Similarly, with the UN Decade on Restoration starting next year, leveraging people power for large-scale tree-planting projects will be essential, and if well-managed with biodiversity in mind, could be a valuable chance for public engagement and a rare ecological good news story. 
Published online: 28 October 2020

https://doi.org/10.1038/s41559-020-01345-4

\section{References}

1. Derryberry, E. P., Phillips, J. N., Derryberry, G. E., Blum, M. J. \& Luther, D. Science https://doi.org/10.1126/science.abd5777 (2020).

2. Rutz, C. et al. Nat. Ecol. Evol. 4, 1156-1159 (2020).
3. Amano, T., Lamming, J. D. L. \& Sutherland, W. J. BioScience 66, 393-400 (2016).

4. Soga, M. \& Gaston, K. J. Front. Ecol. Environ. 14, 94-101 (2016).

5. Pandya, R. E. Front. Ecol. Environ. 10, 314-317 (2012).

6. Bailey, K., Morales, N. \& Newberry, M. III Nat. Ecol. Evol. 4, 1294-1295 (2020).

7. Saunders, M. E. et al. Austral Ecol. 43, 635-642 (2018)

8. Mitchell, N. et al. PLoS ONE 12, e0186285 (2017).
9. Redding, D. W. et al. Nature 571, 103-106 (2019).

10. Reynolds, M. D. et al. Sci. Adv. 3, el700707 (2017).

11. Moore, M. P., Lis, C., Ghergel, I. \& Martin, R. A. Ecol. Lett. 22, 437-446 (2019).

12. Pocock, M. J. O., Tweddle, J. C., Savage, J., Robinson, L. D. \& Roy, H. E. PLoS ONE 12, e0172579 (2017).

13. Fritz, S. et al. Nat. Sustain. 2, 922-930 (2019). 